\title{
Venom immunotherapy in patients with clonal mast cell disorders: IgG4 correlates with protection
}

\author{
Jesper Jarkvist ${ }^{1,2}$ | Clara Salehi $^{1}$ | Cem Akin ${ }^{3}$ | Theo Gülen ${ }^{1,2,4}$ (D)
}

${ }^{1}$ Department of Respiratory Medicine and Allergy, Karolinska University Hospital Huddinge, Stockholm, Sweden

${ }^{2}$ Immunology and Allergy Unit, Department of Medicine Solna, Karolinska Institutet, and Karolinska University Hospital, Stockholm, Sweden

${ }^{3}$ Division of Allergy and Clinical Immunology, Department of Internal Medicine, University of Michigan Health System, Ann Arbor, MI, USA

${ }^{4}$ Mastocytosis Center Karolinska, Karolinska Institutet and Karolinska University

Hospital, Stockholm, Sweden

\section{Correspondence}

Theo Gulen, Department of Respiratory Medicine and Allergy, K85, Karolinska University Hospital Huddinge, SE-141 86 Stockholm, Sweden.

Email:Theo.Gulen@sll.se

Funding information

Konsul TH C Bergh Foundation, Sweden; The regional agreement on medical training and clinical research (ALF) between Stockholm County Council and Karolinska Institutet.

\begin{abstract}
Background: Patients with clonal mast cell disorders (cMCD), systemic mastocytosis (SM) and monoclonal mast cell activation syndrome (MMAS), represent an increased risk for Hymenoptera venom anaphylaxis (HVA). Lifelong venom immunotherapy (VIT) is recommended; however, its efficacy and safety are controversial. Hence, we sought to evaluate the efficacy and safety of VIT in HVA patients with CMCD.

Methods: A retrospective study was conducted among 46 patients with Vespula venom allergy who had experienced severe HVA, 32 cMCD (22 with SM and 10 with MMAS) and 14 controls. There were no differences between CMCD patients and controls in age (58 vs 66) and duration of VIT (47 vs 48 months), respectively.

Results: During VIT, 11 (34\%) cMCD patients experienced adverse reactions (ARs) (7\% in controls), including 1 anaphylaxis. There were 23 re-stings in 17 (53\%) patients during VIT. Of episodes, four (17\%) presented with anaphylaxis, 14 (60\%) presented with local reaction, and five (23\%) were asymptomatic. In 11 episodes (48\%), the patient did not take epinephrine, of these $8(73 \%)$ presented with local reaction, and $3(27 \%)$ were asymptomatic. Patient-based protection from anaphylaxis was $76 \%$ $(4 / 17)$ in CMCD vs. $100 \%$ in controls during VIT. The venom-specific IgG4 concentrations increased during VIT $(P<.001)$ although tryptase and IgE were unaltered.

Conclusion: Both safety and efficacy of VIT in CMCD patients were slightly reduced than controls. Severe ARs were rare. The elevated IgG4 levels may be a biomarker for efficacy of VIT in CMCD patients, as it correlates with protection from re-stings.
\end{abstract}

\section{KEYWORDS}

D816V mutation, hymenoptera venom anaphylaxis, IgG4, mastocytosis, venom

immunotherapy

\section{1 | INTRODUCTION}

Clonal mast cell disorders (CMCD) comprise systemic mastocytosis (SM) and monoclonal mast cell activation syndrome (MMAS). ${ }^{1,2}$ Common to these two conditions is the presence of mast cell (MC) clonality, as reflected in a mutation in codon 816 of KIT and/or occurrence of immunophenotypically aberrant MCs expressing CD25. ${ }^{3}$ In patients with MMAS, the WHO criteria for SM are not fully met. ${ }^{3}$
Anaphylaxis is a well-known feature of CMCD; particularly, venom allergy represents an increased risk of severe, even fatal, sting anaphylaxis in these patients. ${ }^{4,5}$ Although the overall prevalence of Hymenoptera venom-induced anaphylaxis (HVA) is approximately $25 \%$ in patients with $\mathrm{SM},{ }^{6}$ the underlying reason(s) for this association remains elusive. The aggravated risk of severe HVA might be due to increased MC burden, perivascular aggregation of MCs and an amplified IgE reaction due to the presence of D816V KIT mutation. ${ }^{7}$ These findings stress the importance of 


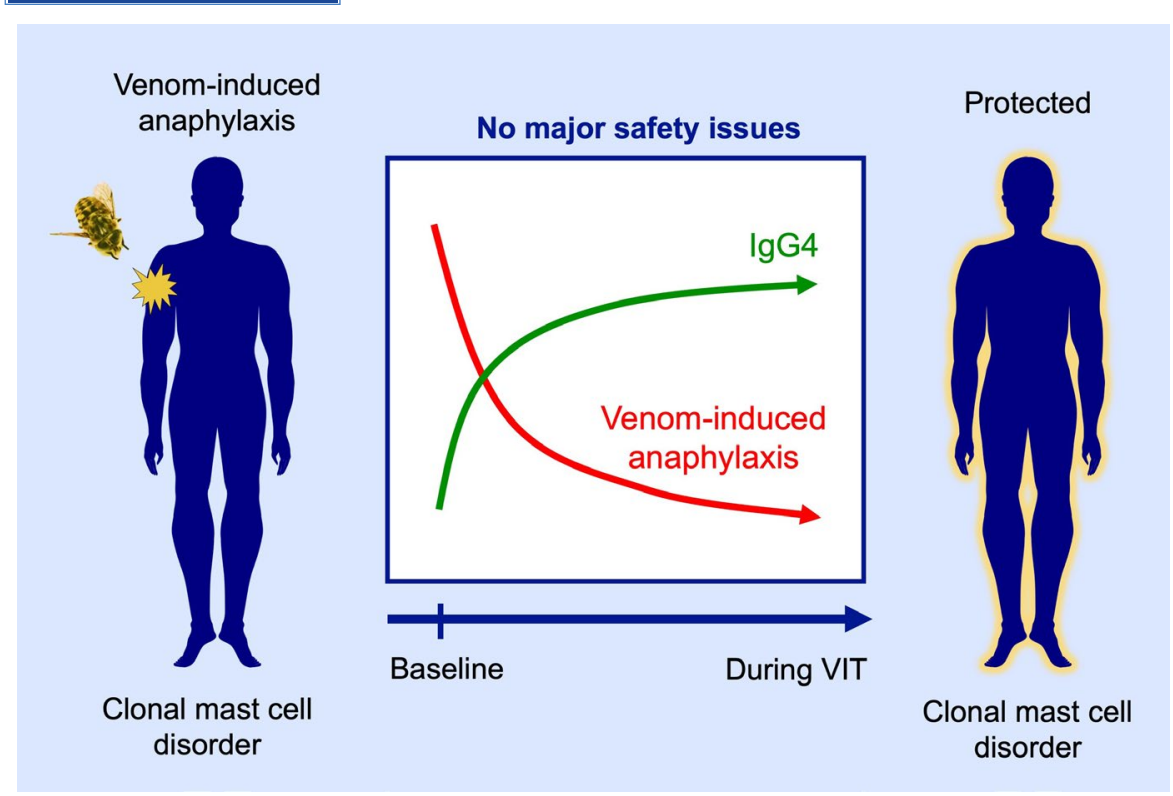

\section{GRAPHICAL ABSTRACT}

Patients with clonal mast cell disorders (CMCD) convey increased risk for venom-induced anaphylaxis and lifelong venom-immunotherapy (VIT) is recommended, when appropriate. VIT appears to be rather safe and effective in cMCD patients, as the risk of severe adverse reactions are rare. Elevated levels of venom-specific IgG4 correlates with protection from re-sting anaphylaxis in CMCD patients during VIT.

accurate diagnostics; therefore, underlying CMCD should be considered in patients with HVA that have elevated serum baseline tryptase levels (sBT) ( $\geq 11.4 \mathrm{ng} / \mathrm{mL})$. Additionally, sensitization against Hymenoptera venom components should be confirmed by skin prick test and/or serum-specific IgE assays. However, it must be noted that patients with CMCD and HVA may lack sensitization to venoms. ${ }^{4,8}$

Venom immunotherapy (VIT) has been used for treatment of patients with diagnosed cMCD and HVA since 1990s; however, increased adverse reactions (ARs) and reduced efficacy have been main concerns in earlier studies. ${ }^{9}$ As many as 6 in 7 patients with CMCD had reactions to field re-stings, despite ongoing VIT treatment. ${ }^{9}$ This raised concerns and controversies about current recommendations regarding necessity and duration of VIT in patients with CMCD. Interestingly, more recent studies found VIT to be safe and effective in patients with CMCD but acknowledged a reduced efficacy and more frequent ARs during the administration of VIT compared with the general population. ${ }^{10-13}$ At present, there is no evidence that VIT induces sustainable tolerance in patients with CMCD. Hence, the current recommendation is to proceed lifelong VIT in these patients. ${ }^{14}$ Additionally, the known markers of successful VIT (IgG4, IL-10, regulatory T cells) have not been specifically studied in CMCD patients.

Thus, there is a continuing unmet need for further studies regarding VIT in patients with CMCD, as the available observations are based on limited number of reports. Here, we sought to determine the safety and efficacy of VIT by evaluating ARs during the administration of VIT and assessing the severity of field re-sting reactions. Furthermore, we also analysed the efficacy by monitoring certain biomarkers before and during ongoing treatment in patients with $\mathrm{CMCD}$.

\section{2 | METHODS}

\section{1 | Patients and clinical procedures}

Between January 2006 and December 2018, 396 consecutive adult patients ( $\geq 18$ years old) have been referred to the Mastocytosis Centre Karolinska due to clinically suspected CMCD including patients with mastocytosis in the skin, patients with severe anaphylaxis or patients with elevated baseline tryptase levels of unknown origin. The final diagnoses, for example, SM or MMAS, were obtained after a comprehensive medical evaluation and bone marrow investigation following WHO criteria. ${ }^{3}$ Moreover, sBT levels (Thermo Fisher) were measured.

Anaphylactic reactions were diagnosed in accordance with NIH clinical criteria, when either reduced blood pressure or associated symptoms such as syncope/presyncope and/or respiratory compromise were present accompanied by the involvement of the skin-mucosal tissue and/or gastrointestinal symptoms. ${ }^{15}$ In cases where assessments were difficult because of insufficient documentation, only patients who had syncope episodes after exposure to a likely trigger (eg insect sting) were assessed to have anaphylaxis. When available, serum tryptase levels during acute episodes were applied to confirm anaphylaxis. The diagnosis of HVA was based on clinical history, skin prick test and/or allergenspecific lgE. ${ }^{16}$ 
Total investigated patients due to suspected mast cell disorder from 2006 to $2018(n=396)$

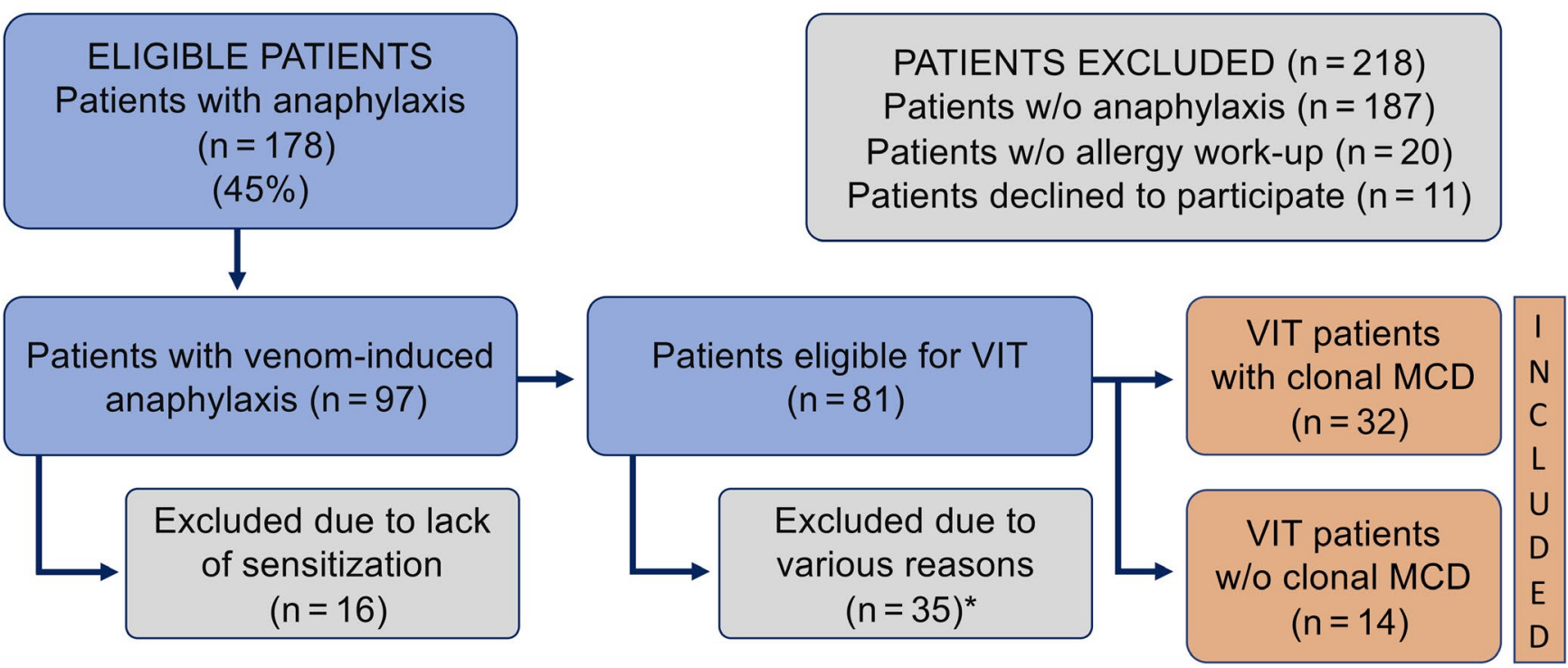

FIGURE 1 The flow chart illustrates the selection process of the study subjects. *35 patients were excluded due to various reasons (15 received VIT at other clinics, 7 had elevated baseline tryptase levels without underlying CMCD, 6 declined to undergo VIT, 4 had comorbidities with cancer, two patients were investigated during study start, and 1 patient was sensitized for honeybee only). Abbreviations: w/o, without; VIT, venom immunotherapy; MCD, mast cell disorders

\section{2 | Allergy work-up}

As previously described, ${ }^{4}$ all patients went through a complete allergic work-up at Karolinska University Hospital Huddinge, allergy outpatient clinic including medical history, skin prick testing (SPT) with commercial extracts (ALK-Abelló A/S, Horsholm, Denmark) of standard aeroallergens, food allergens and allergen of Hymenoptera venom (honeybee and common wasp). The venom-specific IgE antibody test for honeybee and Vespula venom (ImmunoCAP Phadiatop ${ }^{\circledR}$, Thermo Fisher, Uppsala, Sweden) was also performed and considered positive for values $>0.10 \mathrm{kU} / \mathrm{L}$. Moreover, serum concentrations of component-specific venom IgE (Ves r5, Ves r1, Api $\mathrm{m} 1$ and Api m10); venom-specific IgG4; and serum total IgE were determined by ImmunoCAP ${ }^{\circledR}$ (Thermo Fisher).

\section{3 | Study design and subjects}

A retrospective study was conducted. Data were collected through review of electronic patient records. Of 396 investigated patients, 178 had experienced at least one anaphylactic reaction (Figure 1). Among these, 97 patients with HVA were identified. After excluding 51 patients, $46 \mathrm{pa}-$ tients with Vespula venom allergy who fulfilled the criteria for $\mathrm{VIT}^{14}$ were enrolled in this study (Figure 1). Of study subjects, 32 had diagnoses of CMCD and HVA. Additionally, 14 patients with HVA and normal sBT levels ( $<11.4 \mathrm{ng} / \mathrm{mL}$ ) were included as controls to compare the safety and efficacy of VIT. The study was approved by Stockholm's Ethics Review Board (Dnr: 2011/1750/31/3 and Dnr: 2018/2621-31), and all enrolled patients provided their written informed consent to participate.

\subsection{Venom immunotherapy and follow-up}

Venom immunotherapy was started with Vespula extract (ALKAbelló) according to a 7-week traditional build-up schedule at the allergy outpatient clinic. Patients received incremental, weekly doses of depot venom extract subcutaneously until a maintenance dose ( $1 \mathrm{~mL}$ of $100000 \mathrm{SQ}-\mathrm{U} / \mathrm{mL}$, corresponding to $100 \mu \mathrm{g}$ ) was reached. The achieved maintenance dose was then given every four (between May and October; high-risk season) or 6 weeks (between November and April; low-risk season for stings), and follow-up ended on 31 December 2018. All patients received premedication with $\mathrm{HI}$ blockers, 1-2 hours prior to VIT, and were observed 45 minutes after each injection. Extra drugs were given in case of acute reactions. Additionally, simultaneous treatment with omalizumab (Xolair ${ }^{\circledR}$ ) during VIT was documented, when applied.

Blood samples were collected as part of routine patient care, and biological markers were in general analysed before VIT started and at different time points during VIT. Information about possible ARs was documented during the routine VIT visits, or as in few cases, patients reported late ARs by phone. Data about field re-stings during VIT and their outcomes were documented at the time of next follow-up visit and confirmed by the emergency room (ER) reports, when available.

\section{5 | Statistical analysis}

All analyses were performed using IBM SPSS Statistics 24.0 (IBM). Values of $P<0.05$ were considered statistically significant. Frequencies were reported for categorical variables, and group 
differences were analysed by using Fisher's exact test. Continuous variables were presented as median values and ranges. Because the distribution of the data was not normal according to Shapiro-Wilk test, the nonparametric Mann-Whitney $U$ test or Kruskal-Wallis test was used to compare the group distributions, when appropriate. Additionally, when crude data analysis was significant, a post hoc analysis was performed using the Wilcoxon's matched pair rank sum test to detect alterations at different time points within groups. We used Spearman's rank correlation coefficient to demonstrate clinical relevance of venom-specific IgG4 during re-stings. Since re-stings were unpredictable, and it was impossible to assess the IgG4 on the day of re-sting, we used IgG4 concentrations closest to the day of re-sting occasions.

\section{3 | RESULTS}

\section{1 | Patient characteristics and clinical data prior to venom immunotherapy}

Of 32 patients with cMCD, 22 were diagnosed with indolent SM; 64\% were males and had a median age of 61 (range 40-80) at diagnosis. Additionally, $45 \%$ of patients with SM (10/22) had cutaneous involvement, $36 \%$ had MC clusters in bone marrow biopsy, and $86 \%$ carried a KIT D816V mutation. They started VIT at a median age of 62 (40-81) years and received the treatment for a median period of 45 months (7-154). The remaining $10 \mathrm{cMCD}$ patients were diagnosed with MMAS; $56 \%$ were males and had a median age of 55 (range 38-71). All MMAS patients had MCs expressing CD25+ aberrant immunophenotype, whereas $22 \%$ concomitantly carried a KIT D816V mutation. They started VIT at a median age of 52 (38-66) years and received VIT for a median period of 77 months (9-104). Bone marrow examinations were offered to all but performed only in 4 of 14 controls, since most refused to undertake the procedure. Additionally, peripheral blood KIT D816V mutation was negative in all analysed controls $(n=11)$.

Subjects with cMCD were more often males (63\%) than in the control group (43\%), although this finding was not statistically significant (Table 1). Serum baseline tryptase levels were significantly higher in patients with $\mathrm{CMCD}$, whereas total IgE were significantly higher in controls (Table 1). Moreover, controls presented with significantly higher levels of venom-specific lgE and component rVes v5 ( $P=.001$; Table 1 ). To note, $57 \%$ of patients in control group suffered from cardiovascular comorbidities including hypertension and/or angina pectoris prior to the culprit sting reaction $(P<.001$; Table 1$)$. Regarding the severity of culprit reactions, both $\mathrm{CMCD}$ patients and controls presented with severe HVA, where syncope frequently occurred (82\% of the patients with SM, 56\% with MMAS patients and 57\% in controls). Additionally, controls frequently presented with skin symptoms $(P<.001)$; otherwise, there were no significant differences between the two groups in terms of reaction severity (Figure 2).

VIT started in subjects with CMCD at an earlier age with a median of 58 years compared to 66 years in controls (Table 2). The duration of VIT was similar between the two groups and varied among individual patients due to the retrospective nature of the study. A
TAB LE 1 Comparison of demographic, clinical and laboratory characteristics of CMCD patients with HVA compared to controls with HVA prior to VIT

\begin{tabular}{|c|c|c|c|}
\hline $\begin{array}{l}\text { Total, } n=46 \\
\text { Age } \geq 18 y\end{array}$ & $\begin{array}{l}\text { Clonal MCD } \\
(\mathrm{n}=32)\end{array}$ & $\begin{array}{l}\text { Controls } \\
(n=14)\end{array}$ & $P$-value \\
\hline Male gender, n (\%) & $20 / 32(63)$ & 6/14 (43) & $.333^{\mathrm{a}}$ \\
\hline $\begin{array}{l}\text { Age at diagnosis, me- } \\
\text { dian (range) }\end{array}$ & $59(38-80)$ & $66(46-78)$ & $.129^{\mathrm{b}}$ \\
\hline $\begin{array}{l}\text { sBT levels (ng/mL), } \\
\text { median (range) }\end{array}$ & $\begin{array}{l}18(3.2-68) \\
(7 \mathrm{NA})\end{array}$ & $5.3(2.4-11)$ & $<.001^{b}$ \\
\hline $\begin{array}{l}\text { Total IgE }(\mathrm{kU} / \mathrm{L}) \text {, median } \\
\text { (range) }\end{array}$ & $\begin{array}{l}25(2.6-1000) \\
(11 \mathrm{NA})\end{array}$ & $\begin{array}{l}77(28-790) \\
(2 \mathrm{NA})\end{array}$ & $.033^{b}$ \\
\hline Presence of atopy, $\mathrm{n}(\%)$ & $10 / 32(31)$ & $3 / 14(21)$ & $.724^{a}$ \\
\hline $\begin{array}{l}\text { Positive SPT for wasp, } \\
\mathrm{n}(\%)\end{array}$ & $\begin{array}{l}22 / 31(71) \\
(1 \mathrm{NA})\end{array}$ & $\begin{array}{l}10 / 13(77) \\
(1 \mathrm{NA})\end{array}$ & $1.000^{\mathrm{a}}$ \\
\hline $\begin{array}{l}\text { Positive ImmunoCAP } \\
\text { for wasp, } \mathrm{n}(\%)\end{array}$ & $\begin{array}{l}26 / 28(93) \\
(4 \mathrm{NA})\end{array}$ & $\begin{array}{l}12 / 12(100) \\
(2 \mathrm{NA})\end{array}$ & $1.000^{\mathrm{a}}$ \\
\hline $\begin{array}{l}\text { Wasp-specific lgE } \\
\text { (kU/L), median (range) }\end{array}$ & $\begin{array}{l}0.54(0.09-48) \\
(10 \mathrm{NA})\end{array}$ & $\begin{array}{l}5.3(0.12-50) \\
(3 \mathrm{NA})\end{array}$ & $.007^{b}$ \\
\hline $\begin{array}{l}\text { Component rVes v } 5 \\
\text { (kU/L), median (range) }\end{array}$ & $\begin{array}{l}0.30(0.1-25) \\
(13 \mathrm{NA})\end{array}$ & $\begin{array}{l}6.2(0.11-60) \\
(1 \mathrm{NA})\end{array}$ & $.001^{\mathrm{b}}$ \\
\hline $\begin{array}{l}\text { Component rVes } \vee 1 \\
(\mathrm{kU} / \mathrm{L}) \text {, median (range) }\end{array}$ & $\begin{array}{l}0.10(0.1-75) \\
(15 \mathrm{NA})\end{array}$ & $\begin{array}{l}0.10(0.1-0.16) \\
(11 \mathrm{NA})\end{array}$ & ND \\
\hline $\begin{array}{l}\text { Wasp-specific lgG } \\
\text { (mg/L), median (range) }\end{array}$ & $\begin{array}{l}0.52(0.04-8.9) \\
(22 \mathrm{NA})\end{array}$ & $\begin{array}{l}2.20 \\
(13 \mathrm{NA})\end{array}$ & ND \\
\hline $\begin{array}{l}\text { Syncope from wasp } \\
\text { sting prior to VIT }\end{array}$ & $24 / 32(75)$ & $8 / 14(57)$ & $.301^{\mathrm{a}}$ \\
\hline $\begin{array}{l}\text { Comorbidity with CVD } \\
\text { prior to first sting, } \\
\mathrm{n}(\%)\end{array}$ & $2 / 32(6)$ & $8 / 14(57)$ & $<.001^{a}$ \\
\hline
\end{tabular}

Abbreviations: $\mathrm{CMCD}$, clonal mast cell disorders; CVD, cardiovascular disease; HVA, Hymenoptera venom anaphylaxis; NA, not analysed; ND, not done; sBT, serum baseline tryptase; SPT, skin prick test; VIT, venom immunotherapy.

${ }^{a} P$-values were calculated using Fisher's exact test;

${ }^{\mathrm{b}} P$-values were calculated using a 2-tailed Mann-Whitney $U$ test; bold indicates statistical significance $(P<.05)$.

total of seven patients discontinued VIT mainly due to the appearance of comorbidities (including cancer) or were referred to home clinics for practical reasons.

\section{2 | Adverse reactions during VIT}

Among patients with cMCD, 11 (34\%) experienced ARs, eight (73\%) during the induction phase and three (27\%) during maintenance. The total number of episodes was 16 , and epinephrine was administered twice (Table 2). Nine of these episodes were only local reactions, and six involved milder systemic reactions (without respiratory/cardiovascular symptoms). Conversely, ARs in controls were limited to one patient (7\%) who reacted with milder systemic reaction (Table 2). Anaphylaxis was observed only in one patient with SM who received simultaneous immunotherapy against wasp and honeybee and occurred nine months after the maintenance phase of VIT had started. The patient presented with flush and general weakness a 
FIGURE 2 Clinical symptoms in patients with $\mathrm{CMCD}$ and controls during the culprit anaphylactic reactions to wasp sting prior to VIT. Statistical analysis performed by Fisher's exact test. *Hypotension, objectively verified. Abbreviations: $\mathrm{F} / \mathrm{U}$, faecal and/or urinary incontinence; $\mathrm{GI}$, gastrointestinal cramps, nausea, vomiting and diarrhoea; RESP, respiratory symptoms; SKIN, local swelling and redness, itching

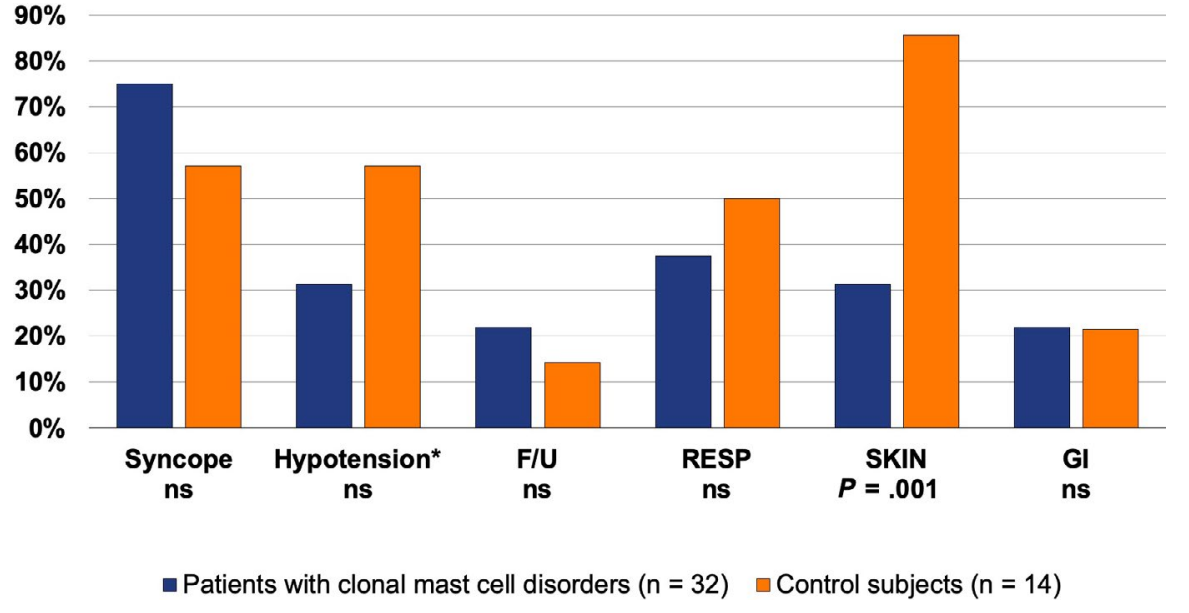

TAB LE 2 Group comparison of adverse reactions during VIT

\begin{tabular}{|c|c|c|c|}
\hline & $\begin{array}{l}\text { Clonal MCD } \\
(\mathrm{n}=32)\end{array}$ & $\begin{array}{l}\text { Controls } \\
(n=14)\end{array}$ & $P$-value \\
\hline Age at start of VIT, median (range) & $58(38-81)$ & $66(45-79)$ & $.127^{\mathrm{a}}$ \\
\hline Total VIT duration (months), median (range) & $47(7-154)$ & $48(13-65)$ & $.277^{\mathrm{a}}$ \\
\hline Patients with adverse reactions from VIT, n (\%) & $11 / 32(34)$ & $1 / 14(7)$ & $.073^{\mathrm{b}}$ \\
\hline Total number of episodes with adverse reactions ( $\mathrm{n}$ ) & 16 & 1 & ND \\
\hline Mild systemic adverse reactions, $\mathrm{n}(\%)$ & $7 / 16(44)$ & $1 / 6(17)$ & ND \\
\hline Patients with anaphylactic reaction from VIT, n (\%) & $1 / 32(3)$ & $0 / 6(0)$ & ND \\
\hline Total number of injections, $\mathrm{n}$ & 1781 & 493 & ND \\
\hline Number of injections needed per adverse reaction, $\mathrm{n}$ & 111 & 1 & ND \\
\hline Number of injections needed per anaphylaxis, $n$ & 1781 & ND & ND \\
\hline Use of adrenaline in adverse reaction, $\mathrm{n}(\%)$ & $2 / 16(18)$ & $0 / 1(0)$ & ND \\
\hline
\end{tabular}

Abbreviations: CMCD, clonal mast cell disorders; ND, not done; VIT, venom immunotherapy. ${ }^{a} P$-values were calculated using a two-tailed Mann-Whitney $U$ test.

${ }^{\mathrm{b}} P$-value was calculated using Fisher's exact test. few minutes after receiving both VIT injections. The blood pressure was initially normal (122/70 mm Hg). Despite receiving immediate treatment with epinephrine, 3-4 minutes later the patient had documented hypotension (80/44 $\mathrm{mm} \mathrm{Hg}$ ). He received another dose of epinephrine and intravenous fluid before he was transferred to the ER. Afterwards, VIT could be resumed, however, solely with wasp extract and in conjunction with omalizumab (Xolair@) treatment (300 mg q2weeks). The omalizumab treatment has been continued, and no further incidences occurred since then. Omalizumab protection was applied in two other patients (diagnosed with SM and MMAS, respectively), and the treatment was discontinued after 54 and 20 months, respectively. Thereby, VIT was tolerated, as these patients still receive VIT. We needed to apply omalizumab in these patients to achieve maintenance doses since it has been reported in the literature with successful results to allow administration of VIT.

\section{3 | Protection from re-sting reactions while ongoing VIT}

A total of 17 (53\%) patients with cMCD were re-stung in 23 separate episodes. Epinephrine was used in 12 episodes. One sting occurred during the induction phase and the remaining during the maintenance phase. Six episodes were asymptomatic, 10 resulted in local reaction, and three resulted in milder systemic reactions. Four episodes (in four separate patients) were assessed to be anaphylactic reactions, and all four patients used intramuscular epinephrine and sought emergency care (Table 3). Table S1 shows the main characteristics of 5 patients with anaphylaxis during VIT (four anaphylaxis during re-stings and one anaphylaxis as AR). Interestingly, these five patients had positive SPT for wasp at baseline (Table S1), whereas only 22 patients in overall cohort (71\%) (Table 1). Nevertheless, this was not clinically significant. Meanwhile, five $(35 \%)$ controls were re-stung in a total of eight episodes, which occurred during the maintenance phase of VIT (Table 3). Epinephrine was used in two episodes, although no anaphylaxis was observed.

During VIT, clinical symptoms from re-stings were found to be less severe compared to culprit reactions. Most re-sting reactions in patients with cMCD were limited only to skin; moreover, $23 \%$ were asymptomatic. In contrast to the culprit reactions, where $75 \%$ of patients with $\mathrm{CMCD}$ had syncope, only one patient $(5 \%)$ had syncope during re-stings $(P<.001)$. Consequently, we observed 76\% (13/17) patientbased and $83 \%(19 / 23)$ episode-based protection from anaphylaxis in patients with $\mathrm{CMCD}$ during field re-sting reactions $(P<.001)$. 


\begin{tabular}{|llll|}
\hline & $\begin{array}{l}\text { Clonal MCD } \\
(\mathbf{n}=32)\end{array}$ & $\begin{array}{l}\text { Controls } \\
(\mathbf{n}=14)\end{array}$ & P-value \\
\hline Patients who were re-stung during VIT, $\mathrm{n}(\%)$ & $17 / 32(53)$ & $5 / 14(35)$ & .346 \\
\hline Number of patients with anaphylactic reaction, $\mathrm{n}(\%)$ & $4 / 17(24)$ & $0(0)$ & ND \\
\hline Protection from anaphylaxis per subject, $\mathrm{n}(\%)$ & $13 / 17(76)$ & $0 / 5(100)$ & $\mathrm{ND}$ \\
\hline Number of episodes of re-stings (n) & 23 & 8 & ND \\
\hline Episodes with anaphylactic reaction to re-sting, $\mathrm{n}(\%)$ & $4 / 23(17)$ & $0 / 8(0)$ & ND \\
\hline Protection from anaphylaxis per episode, $\mathrm{n}(\%)$ & $19 / 23(83)$ & $8 / 8(100)$ & ND \\
\hline Use of adrenaline in episode of re-sting, $\mathrm{n}(\%)$ & $12 / 23(52)$ & $1 / 8(13)$ & .095 \\
\hline
\end{tabular}

TABLE 3 Comparison of patients with CMCD and control subjects regarding resting reactions during $\mathrm{VIT}$

Abbreviations: $\mathrm{CMCD}$, clonal mast cell disorder; ND, not done; VIT, venom immunotherapy.

$P$-values were calculated using Fisher's exact test.

\subsection{Dynamics of biomarkers in patients with cMCD during VIT}

Serum concentration of wasp-specific lgG4 increased significantly in patients with CMCD over the period of VIT ( $P<.001$; Table S2). In contrast, repeated measures of other biomarkers including plasma levels of sBT, total IgE, wasp venom-specific IgE or venom component rVes v5 did not show significant dynamics during VIT compared to baseline levels. Calculation of specific ratios incorporating lgG4 did not provide any additional significance beyond lgG4 levels alone (Figure S2).

On group level, the median of wasp-specific lgG4 before VIT was $0.52 \mathrm{mg} / \mathrm{L}$ (range 0.04-8.9) in CMCD (Table S2). Two outliers were identified (2.4 and $8.9 \mathrm{mg} / \mathrm{L}$, respectively) at baseline, and both previously completed a 5-year VIT course (Figure 3A). Afterwards, VIT was restarted when both patients were diagnosed with $C M C D$. We also evaluated wasp-specific lgG4 levels in six CMCD patients in relation to nine re-sting reactions and found an inverse correlation between IgG4 concentrations and reaction severity $(P<.01$; Figure 3B). Since $\lg G 4$ could not be obtained on the day of the re-stings, we analysed the closest IgG4 values in relation to day of reactions (median 4 months, range $0-10$ ). Two patients who were asymptomatic in three episodes when re-stung had wasp-specific lgG4 levels between 21 and $25 \mathrm{mg} / \mathrm{L}$. Conversely, the only patient who reacted in two episodes during the same summer with mild systemic reactions presented with lower wasp-specific IgG4 levels, 10 and $11 \mathrm{mg} / \mathrm{L}$ prior to and after the stings. The remaining five patients who only experienced local reactions $(<10 \mathrm{~cm}$ in diameter) had IgG4 concentrations between 8 and $21 \mathrm{mg} / \mathrm{L}$.

\section{4 | DISCUSSION}

Our results support that VIT appears to be rather safe and effective in patients with CMCD and HVA as the risk of severe systemic ARs was rare. Additionally, significantly increased serum concentrations of wasp-specific lgG4 were detected; this is, to our knowledge, the first report demonstrating this phenomenon in CMCD patients.

While allergic reactions to foods and drugs have been reported in $\mathrm{CMCD}$, the primary trigger for IgE-mediated severe, even fatal, anaphylactic reactions remains Hymenoptera stings. ${ }^{17,18}$ In general, VIT induces protection from severe sting reactions in HVA patients during and after discontinuation of therapy. ${ }^{19-22}$ Nevertheless, there have been controversies regarding its safety and efficacy in patients with CMCD. ARs to VIT have been reported in $29 \%$ of patients with CMCD compared to $14 \%$ in general HVA population. ${ }^{12,23}$ VIT protocols applied during induction phase are also essential since increased frequencies of ARs were reported in rush- or ultrarush protocols. ${ }^{24}$ Interestingly, no ARs were observed in a recent study of eight mastocytosis patients receiving VIT by ultrarush protocol. ${ }^{25}$ Our study supports previous findings as we found a fivefold increased risk for ARs in CMCD patients compared to controls (34\% vs $7 \%$, respectively). Additionally, the ARs occurred mainly during the build-up phase, of which $44 \%$ were milder systemic reactions. Only one patient suffered from an anaphylactic reaction (3\%). Of note, this patient was treated with both honeybee and wasp venom simultaneously, a procedure reported to increase the risk of ARs. ${ }^{12}$ No patients in the current study had to be discontinued; however, VIT in patients with CMCD is not risk-free.

Efficacy of VIT is typically evaluated by sting challenges and reports from field re-stings; however, sting challenges are not performed in all clinics. ${ }^{14}$ Several studies have reported on efficacy as the rate of protection from systemic reactions; nevertheless, no universally accepted grading system exists to classify the severity of systemic reactions. ${ }^{26}$ This complicates comparison of the efficacy of VIT across different studies. ${ }^{27}$ In patients with mastocytosis, the protection rate varied from $14 \%$ to $85 \%$ in a review of 10 studies with 201 patients. ${ }^{12}$ Our results support VIT being an efficient treatment to prevent anaphylaxis, since only four episodes (17\%) from field restings were classified as anaphylaxis. That implies a protection rate of $83 \%$, in line with a previous report (86\%). ${ }^{10}$ Only one patient with CMCD reacted with syncope when re-stung compared to $75 \%$ during culprit reactions $(P<.001)$. There were no fatalities in our series. However, since this protection can only be sustained during VIT, the current guidelines recommend lifelong VIT in patients with CMCD.

Notably, the severity of culprit reactions in patients with CMCD appears to be independent from concomitant cardiovascular diseases (CVDs) and use of beta blockers and/or ACE inhibitors since the control subjects more frequently presented with CVDs $(P<.001)$ without experiencing more severe reactions. Thus, the 


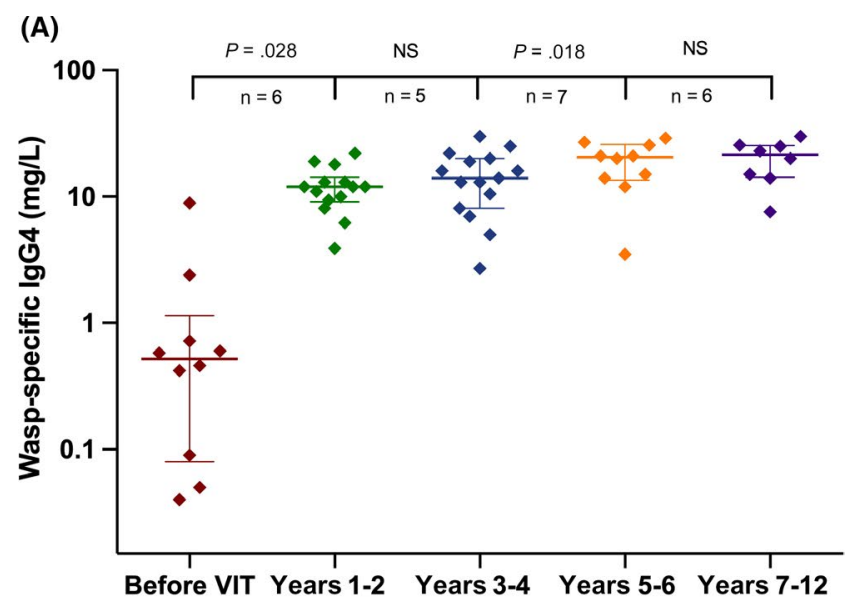

(B)

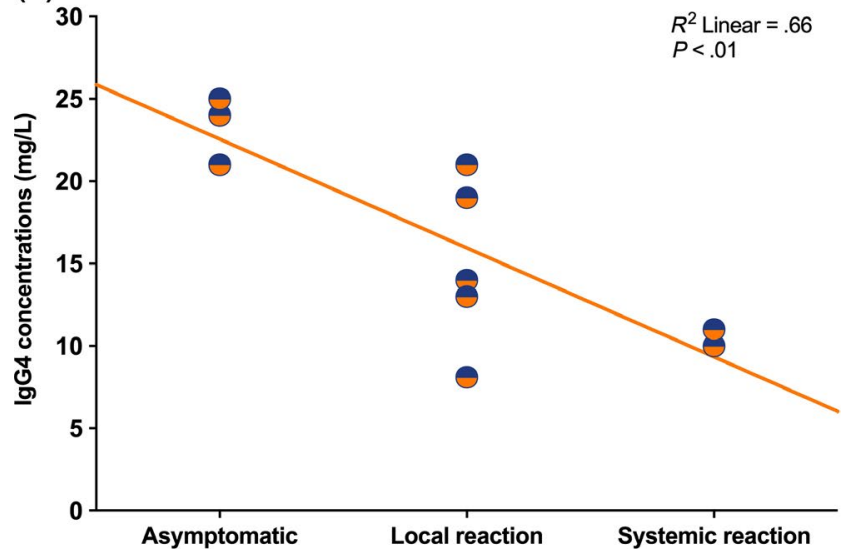

FIGURE 3 A, Dynamic of wasp-specific IgG4 levels in cMCD patients during VIT. Two outliers were identified $(2.4$ and $8.9 \mathrm{mg} / \mathrm{L}$, respectively) at baseline, and both patients previously received VIT. P-values were analysed with Wilcoxon's matched pair rank sum test. A significant increase was noted between baseline and years 1-2 $(P=.028)$ and between years 3-4 and years 5-6 $(P=.018)$. $\mathrm{n}$; number of matched patients at different time points. Different colours represent different time periods. B, Correlation between wasp-specific IgG4 levels and severity of re-sting reaction. The reactions were classified as asymptomatic, local reactions $(<10 \mathrm{~cm}$ in diameter) and mild systemic reactions (without respiratory or circulatory compromise). Correlation was quantified by using Spearman's rank correlation coefficient

mechanisms leading to severe HVA in CMCD patients may be different from those of controls; for instance, they might be due to the inherent mast cell hyperreactivity. Additionally, the serum concentration of the venom-specific IgE and component-specific venom IgE rVes v5 (which is the dominating allergic epitope in Sweden) levels at baseline was lower in patients with CMCD compared to controls $(P=.013)$. It is known that patients with SM typically exhibit lower levels of total and specific IgE, presumably due to the adsorption of specific IgE by the expanded MC burden ${ }^{28}$; however, whether this is true also for $r$ Ves $v 5$ has not been previously investigated.

The immunological mechanisms underlying VIT efficacy have not been fully elucidated, although induction of peripheral tolerance and the generation of allergen-specific regulatory $T$ (Treg) and B (Breg) cells appear to be cardinal features. Treg cells are characterized by IL-10 secretion that directly or indirectly suppresses effector cells including mast cells, and also have influence on B cells, suppressing IgE production and inducing the production of blocking-type lgG4 antibodies against venom allergens. ${ }^{29-33}$ The earlier studies concerned patients from the general HVA population and acknowledged that specific IgG levels increased significantly during VIT but also decreased significantly when VIT was discontinued. ${ }^{21,34-36}$ Since a protective effect was still evident even after VIT was discontinued, they concluded other immunological mechanisms rather than specific lgG were likely responsible. Nevertheless, these studies did not involve the IgG subclass, IgG4. Interestingly, another investigation reported that specific honeybee IgG4 concentrations remained increased two years after discontinuing VIT against honeybee, suggesting a long-lasting protection of specific IgG4. ${ }^{37}$ This issue has been re-evaluated in a recent study in patients with HVA and demonstrated increased levels of wasp-specific IgG4 during VIT course, but concentrations declined substantially at 3- and 8-year follow-up after discontinuation of VIT. ${ }^{38}$ Additionally, Golden et $\mathrm{al}^{21}$ found that $88 \%$ of patients who reacted systemically to a sting during VIT had venom-specific IgG antibody levels $\leq 3 \mathrm{mg} / \mathrm{L}$, thereby recommended that monitoring slgG levels during VIT might be predicting residual risk of systemic reactions after a sting. Similarly, a later study reported that monitoring VIT efficacy was only possible in vespid-venom allergy, and the authors proposed that slgG4 threshold for rVes $v 5$ had the highest sensitivity to confirm tolerance. ${ }^{39}$

Currently, no studies have demonstrated sustained tolerance development in patients with $\mathrm{CMCD}$. Because severe or fatal anaphylactic reactions with re-stings occur only in patients with $\mathrm{CMCD}$ after discontinuation of VIT, the immunological mechanism behind clinical efficacy may differ from nonclonal population. ${ }^{12,14}$ However, the dynamic of IgG4 during VIT has never been analysed in patients with CMCD. We demonstrated that venom-specific IgG4 levels constantly increased during VIT and reached a 20 -fold increase during the first 2 years of treatment (Figure 3A). Additionally, when we observed wasp-specific IgG4 in relation to re-sting reactions, we found an inverse correlation between IgG4 levels and reaction severity suggesting that IgG4 concentrations might reflect the clinical efficacy (Figure 3B). Furthermore, we also identified two patients who had anaphylaxis when re-stung, 4 and 11 years after having discontinued VIT. Both patients were later diagnosed with CMCD. The serum concentrations of wasp-specific IgG4 were clearly reduced in both (8.9 and $0.72 \mathrm{mg} / \mathrm{L}$, respectively) before VIT was restarted compared to patients with CMCD who had been continued VIT (median IgG4 concentration $20.5 \mathrm{mg} / \mathrm{L}$ at years $5-6$ ). Hence, treatment failure may be related to inadequate levels of IgG4. VIT could be administered more frequently in these patients to attempt to raise the serum concentration of venom-specific lgG4, thereby increasing efficacy.

Conversely, no significant alterations during different time points of VIT were observed regarding the levels of tryptase, total IgE, wasp venom-specific IgE and component-specific venom IgE. Notably, previous studies demonstrated both decreased and unchanged levels of venom-specific IgE during VIT. ${ }^{23,40,41}$ In the study by Gonzales et $\mathrm{al}^{23}$ with SM patients and HVA, venom-specific IgE 
levels decreased for the entire group during VIT. However, only 6 of 21 patients had Vespula venom allergy. ${ }^{23}$ Remarkably, their patients had relatively high median levels of specific IgE before VIT compared to our patients ( $4.15 \mathrm{KU} / \mathrm{L}$ vs $0.54 \mathrm{kU} / \mathrm{L}$ ). These might be contributing to the contradictory results. Meanwhile, tryptase levels remained unchanged during VIT in both studies. ${ }^{23}$

The main strength of this single-centre study was the homogeneity of the subjects enrolled, since all patients received VIT against wasp, with extract from the same manufacturer and followed by similar VIT protocols. We could therefore compare patients with CMCD to control patients in the same clinical settings. By contrast, paucity of study subjects and retrospective nature of the study lacking relevant data from all patients at all time points analysed were our limitations making difficult to generalize results. When reactions to re-stings were assessed, patient-reported data were unavoidably used and could include a recall bias. Use of epinephrine is a confounding factor that could not be avoided. Finally, several symptoms from systemic allergic reactions and panic attacks overlap, for example anxiety and tachycardia, and all these factors complicate clinicians' assessment of reactions.

In conclusion, our results suggest that both efficacy and safety of VIT are somewhat less reliable in CMCD patients, but the overwhelming benefit justifies the relatively small increase in risk, as severe ARs are rare. The patient-based and episode-based protective rate from field re-sting anaphylaxis were $76 \%$ and $83 \%$, respectively; nevertheless, the efficacy of treatment was only measured during VIT course. Therefore, we support the notion that patients with CMCD should continue VIT indefinitely. Over the course of VIT, venom-specific IgG4 antibodies were increased significantly in patients with CMCD and an inverse correlation between IgG4 levels and reaction severity to field re-stings appears to exist. Using waspspecific IgG4 to monitor clinical efficacy may allow us to schedule more individualized administration of VIT; however, this issue needs to be further explored.

\section{ACKNOWLEDGMENTS}

This study was supported by grants from the Konsul TH C Bergh Foundation, Sweden, and through the regional agreement on medical training and clinical research (ALF) between Stockholm County Council and Karolinska Institutet.

\section{CONFLICT OF INTEREST}

T. Gülen has received lecture fees from Thermo Fisher. C. Akin has received consultancy fees from Blueprint Medicines and Novartis and has a patent for LAD2 cells. J. Jarkvist and C. Salehi declare no relevant conflicts of interest.

\section{AUTHOR CONTRIBUTIONS}

$J$ took active part in the acquisition, analysis and interpretation of the data, and drafting and revising of the manuscript. CS took active part in the acquisition and analysis of the data. CA analysed and interpreted the data and revised the manuscript critically. TG conceptualized and designed the study; collected, analysed and interpreted the data; and wrote and revised the manuscript. All authors approved the final submitted manuscript.

\section{ORCID}

Theo Gülen (iD https://orcid.org/0000-0002-1683-8882

\section{REFERENCES}

1. Akin C. Mast cell activation disorders. J Allergy Clin Immunol Pract. 2014;2:252-257.

2. Akin C. Mast cell activation syndromes. J Allergy Clin Immunol. 2017;140:349-355.

3. Gulen T, Hagglund H, Dahlen B, Nilsson G. Mastocytosis: the puzzling clinical spectrum and challenging diagnostic aspects of an enigmatic disease. J Intern Med. 2016;279:211-228.

4. Gulen T, Hagglund H, Dahlen B, Nilsson G. High prevalence of anaphylaxis in patients with systemic mastocytosis - a single-centre experience. Clin Exp Allergy. 2014;44:121-129.

5. Elberink J, deMonchy J, Kors JW, vanDoormaal JJ, Dubois A. Fatal anaphylaxis after a yellow jacket sting, despite venom immunotherapy, in two patients with mastocytosis. J Allergy Clin Immunol. 1997;99:153-154.

6. Gulen T, Ljung C, Nilsson G, Akin C. Risk factor analysis of anaphylactic reactions in patients with systemic mastocytosis. J Allergy Clin Immunol Pract. 2017;5:1248-1255.

7. Castells MC, Hornick JL, Akin C. Anaphylaxis after hymenoptera sting: is it venom allergy, a clonal disorder, or both? J Allergy Clin Immunol Pract. 2015;3:350-355.

8. Gulen T, Akin C. Pre-emptive evaluation of venom allergy in a patient with systemic mastocytosis. Acta Derm Venereol. 2018;98:149-150.

9. Dubois AE. Mastocytosis and hymenoptera allergy. Curr Opin Allergy Clin Immunol. 2004;4:291-295.

10. Bonadonna P, Zanotti R, Caruso B, et al. Allergen specific immunotherapy is safe and effective in patients with systemic mastocytosis and Hymenoptera allergy. J Allergy Clin Immunol. 2008;121:256-257.

11. Bonadonna P, Gonzalez-de-Olano D, Zanotti R, et al. Venom immunotherapy in patients with clonal mast cell disorders: efficacy, safety, and practical considerations. J Allergy Clin Immunol Pract. 2013;1:474-478.

12. Niedoszytko M, Bonadonna P, Oude Elberink JN, Golden DB. Epidemiology, diagnosis, and treatment of Hymenoptera venom allergy in mastocytosis patients. Immunol Allergy Clin North Am. 2014;34:365-381.

13. Verburg M, Oldhoff JM, Klemans RJ, et al. Rush immunotherapy for wasp venom allergy seems safe and effective in patients with mastocytosis. Eur Ann Allergy Clin Immunol. 2015;47:192-196.

14. Sturm GJ, Varga E-M, Roberts G, et al. EAACl guidelines on allergen immunotherapy: hymenoptera venom allergy. Allergy. 2018;73:744-764.

15. Sampson HA, Muñoz-Furlong A, Campbell RL, et al. Second symposium on the definition and management of anaphylaxis: summary report-second National Institute of Allergy and Infectious Disease/ Food Allergy and Anaphylaxis Network symposium. Ann Emerg Med. 2006;47:373-380.

16. BiloBM, RueffF, Mosbech H, Bonifazi F, Oude-Elberink JN. Diagnosis of hymenoptera venom allergy. Allergy. 2005;60:1339-1349.

17. Carter MC, Metcalfe DD, Matito A, et al. Adverse reactions to drugs and biologics in patients with clonal mast cell disorders: A Work 
Group Report of the Mast Cells Disorder Committee, American Academy of Allergy, Asthma \& Immunology. J Allergy Clin Immunol. 2019;143(3):880-893.

18. Carter MC, Ruiz-Esteves KN, Workman L, Lieberman P, PlattsMills T, Metcalfe DD. Identification of alpha-gal sensitivity in patients with a diagnosis of idiopathic anaphylaxis. Allergy. 2018;73(5):1131-1134.

19. Golden DB. Long-term outcome after venom immunotherapy. Curr Opin Allergy Clin Immunol. 2010;10:337-341.

20. Golden DB. Insect sting allergy and venom immunotherapy. Ann Allergy Asthma Immunol. 2006;96(2 Suppl 1):S16-521.

21. Lerch E, Muller UR. Long-term protection after stopping venom immunotherapy: results of re-stings in 200 patients. J Allergy Clin Immunol. 1998;101:606-612.

22. Dhami S, Zaman H, Varga E-M, et al. Allergen immunotherapy for insect venom allergy: a systematic review and meta-analysis. Allergy. 2017;72:342-365.

23. Gonzales de Olano D, Álvarez-Twose I, Esteban-López MI, et al. Safety and effectiveness of immunotherapy in patients with indolent systemic mastocytosis presenting with Hymenoptera venom anaphylaxis. J Allergy Clin Immunol. 2008;121:519-526.

24. Ruëff F, Przybilla B, Biló MB, et al. Predictors of side effects during the buildup phase of venom immunotherapy for Hymenoptera venom allergy: the importance of baseline serum tryptase. J Allergy Clin Immunol. 2010;126(1):105-111.

25. Gruzelle V, Ramassamy M, Bulai Lidiveanu C, Didier A, Mailhol C, Guilleminault L. Safety of ultra-rush protocols for hymenoptera venom immunotherapy in systemic mastocytosis. Allergy. 2018;73(11):2260-2263.

26. Cox LS, Sanchez-Borges M, Lockey RF. World Allergy Organization systemic allergic reaction grading system: is a modification needed? J Allergy Clin Immunol Pract. 2017;5(1):58-62.

27. Nelson HS, Calderon MA, Bernstein DI, et al. Allergen immunotherapy clinical trial outcomes and design: working toward harmonization of methods and principles. Curr Allergy Asthma. 2017;17:18.

28. Vos B, van Anrooij B, van Doormaal JJ, Dubois A, Oude Elberink J. Fatal anaphylaxis to yellow jacket stings in mastocytosis: options for identification and treatment of at-risk patients. J Allergy Clin Immunol Pract. 2017;5:1264-1271.

29. Till S. Mechanisms of immunotherapy and surrogate markers. Allergy. 2011;66(Suppl 95):25-27.

30. Akdis M, Akdis CA. Mechanisms of allergen-specific immunotherapy: multiple suppressor factors at work in immune tolerance to allergens. J Allergy Clin Immunol. 2014;133:621-631.

31. van de Veen W, Stanic B, Yaman G, et al. IgG4 production is confined to human IL-10-producing regulatory $\mathrm{B}$ cells that suppress antigen-specific immune responses. J Allergy Clin Immunol. 2013;131:1204-1212.

32. Boonpiyathad T, Meyer N, Moniuszko M, et al. High-dose bee venom exposure induces similar tolerogenic B-cell responses in allergic patients and healthy beekeepers. Allergy. 2017;72(3):407-415.
33. Ozdemir C, Kucuksezer UC, Akdis M, Akdis CA. Mechanisms of immunotherapy to wasp and bee venom. Clin Exp Allergy. 2011;41(9):1226-1234.

34. Golden DB, Lawrence ID, Hamilton RH, Kagey-Sobotka A, Valentine MD, Lichtenstein LM. Clinical correlation of the venom-specific IgG antibody level during maintenance venom immunotherapy. J Allergy Clin Immunol. 1992;90:386-393.

35. Muller U, Berchtold E, Helbling A. Honeybee venom allergy: results of a sting challenge 1 year after stopping successful venom immunotherapy in 86 patients. J Allergy Clin Immunol. 1991;87:702-709.

36. Keating MU, Kagey-Sobotka A, Hamilton RG, Yunginger JW. Clinical and immunologic follow-up of patients who stop venom immunotherapy. J Allergy Clin Immunol. 1991;88:339-348.

37. Urbanek R, Kemeny DM, Richards D. Sub-class of IgG anti-bee venom antibody produced during bee venom immunotherapy and its relationship to long-term protection from bee stings and following termination of venom immunotherapy. Clin Allergy. 1986;16(4):317-322.

38. Albanesi M, Nico A, Sinisi A, et al. A 13-year real-life study on efficacy, safety and biological effects of Vespula venom immunotherapy. Clin Mol Allergy. 2018;16(1):2.

39. Arzt L, Bokanovic D, Schrautzer C, et al. Immunological differences between insect venom-allergic patients with and without immunotherapy and asymptomatically sensitized subjects. Allergy. 2018;73(6):1223-1231.

40. van Halteren HK, van der Linden PW, Burgers JA, Bartelink AK. Discontinuation of yellow jacket venom immunotherapy: follow-up of 75 patients by means of deliberate sting challenge. J Allergy Clin Immunol. 1997;100(6 Pt 1):767-770.

41. Saulite I, Hoetzenecker W, Guenova E, Schmid-Grendelmeier P, Glatz $M$. Skin test reactivity to hymenoptera venom after venom immunotherapy correlates inversely with the IgG/IgE Ratio. Int Arch Allergy Immunol. 2017;174(3-4):190-199.

\section{SUPPORTING INFORMATION}

Additional supporting information may be found online in the Supporting Information section at the end of the article.

How to cite this article: Jarkvist J, Salehi C, Akin C, Gülen T. Venom immunotherapy in patients with clonal mast cell disorders: IgG4 correlates with protection. Allergy. 2020;75:169-177. https://doi.org/10.1111/all.13980 